\title{
Balkanologie
}

Balkanologie Revue d'études pluridisciplinaires

Vol. XI, $n^{\circ}$ 1-2 | 2008

Volume XI Numéro 1-2

\section{Migration et identités parmi les Turcs de Bulgarie établis en Turquie (1989-2004)}

\section{Mila Maeva}

Traducteur : Miladina Monova

\section{OpenEdition}

\section{Journals}

Édition électronique

URL : http://journals.openedition.org/balkanologie/1052

DOI : 10.4000/balkanologie.1052

ISSN : 1965-0582

Éditeur

Association française d'études sur les Balkans (Afebalk)

\section{Référence électronique}

Mila Maeva, «Migration et identités parmi les Turcs de Bulgarie établis en Turquie (1989-2004) », Balkanologie [En ligne], Vol. XI, n 1-2 | 2008, mis en ligne le 31 décembre 2008, consulté le 17 décembre 2020. URL : http://journals.openedition.org/balkanologie/1052 ; DOI : https://doi.org/ 10.4000/balkanologie.1052

Ce document a été généré automatiquement le 17 décembre 2020.

(c) Tous droits réservés 


\title{
Migration et identités parmi les Turcs de Bulgarie établis en Turquie (1989-2004)
}

\author{
Mila Maeva
}

Traduction : Miladina Monova

\section{NOTE DE L'ÉDITEUR}

Traduit du bulgare par Miladina Monova

1 La minorité turque en Bulgarie attire depuis longtemps l'attention des chercheurs bulgares. Apparue avec la dissolution de l'Empire ottoman et la formation d'un Etatnation bulgare indépendant (1878), elle représente aujourd'hui 10\% de la population du pays $^{1}$. Du fait de leur nombre important, de leur appartenance ethnique et religieuse ainsi que de leur emplacement géographique, les Turcs bulgares ont toujours fait l'objet d'un traitement politique particulier, aussi bien de la part de l'Etat bulgare que de l'Etat turc. Mais à la différence d'autres recherches effectuées sur la question, la présente étude propose de porter l'attention non sur les Turcs en Bulgarie, mais sur ceux qui ont quitté le pays pour s'établir définitivement en Turquie ${ }^{2}$. L'exode massif de la population turque de Bulgarie s'est effectué en plusieurs temps et cela est dû aux politiques opaques et contradictoires conduites durant plus d'un siècle par les gouvernements bulgare et turc. Ainsi s'est constituée une masse importante d'immigrants originaires de Bulgarie, aux trajectoires complexes et dont les caractéristiques ne pourraient pas être exposées ici de manière exhaustive. Dans cette étude, il sera question essentiellement de ceux qui arrivent en Turquie dans la période 1989-2004, c'est-à-dire après la chute du régime communiste.

Ce que l'on appelle la «nouvelle émigration» des Turcs bulgare vers la Turquie commence à l'été 1989. Elle est due tout d'abord à la campagne de "bulgarisation » des noms et des prénoms qui débute pendant l'hiver 1984 et vise à faire disparaître tous les 
noms d'origine turque et arabe dans le pays. Le gouvernement bulgare introduit également des mesures de restriction des libertés individuelles : interdiction de parler turc dans les lieux publics, de porter des habits traditionnels musulmans, de pratiquer les coutumes et les rituels musulmans tels que la fête du Kurban-Bajram, la circoncision, le mariage traditionnel. Il est même défendu d'écouter et de danser sur de la musique turque. L'Etat bulgare interdit aussi les publications en langue turque ${ }^{3}$. La plateforme idéologique visant à justifier cette politique de "bulgarisation " n'a été formulée que dans l'année 1988, vers la fin de la campagne connue sous le nom de «Processus de renaissance » (văzroditelen proces). L'idéologie officielle se réfère ainsi à l'idée courante d'une "Renaissance manquée " parmi les Turcs bulgares, lesquels, Bulgares d'origine, auraient été assimilés de force à l'identité turque et musulmane au cours des siècles de "joug turc». Aujourd'hui, ils doivent retrouver leur véritable identité. Le but est de construire " une nation socialiste bulgare unie "4. Cette tentative d'assimilation par la violence a conduit, au cours du printemps et de l'été 1989, à une immense vague d'émigration de Turcs de Bulgarie, appelée "la Grande excursion ». Plus de 350000 Turcs ont été forcés à quitter la Bulgarie ${ }^{5}$.

Même si environ 152000 personnes retournent en Bulgarie dès l'année suivante, l'émigration vers la Turquie se poursuit aussi après la chute du régime communiste en novembre 1989. La nouvelle vague est due, cette fois-ci, à la crise économique. Au cours de l'année 1991-1992, les partants sont plus nombreux que ceux de $1989^{6}$. Dans la période de 1990 à 1997, en moyenne entre 30000 et 60000 personnes se rendent en Turquie, munies de visas de séjour temporaire ou de tourisme ${ }^{7}$. Cette vague comprend également ceux qui pendant le régime communiste n'étaient pas autorisés à quitter le pays, ainsi que des personnes qui rejoignent des parents partis avec «la Grande excursion » et déjà établis en Turquie. Certains ne quittent pas le pays avant l'été 1989 parce que leurs enfants sont soit à l'armée, soit encore scolarisés. Ainsi raconte un père de famille : «Au début, je ne suis pas parti parce que les enfants étaient à l'école. Mon, fils aîné étudiait dans une Ecole d'études supérieures en informatique à Kardzhali. Je me suis dit, attendons au moins qu'il termine. Mais après la troisième année je me suis dit, et bien, il peut terminer ses études aussi en Turquie. J'avais appris qu'il pouvait continuer ses études là-bas $»^{8}$.

\section{I - Les motivations de l'émigration}

4 Les dispositions psychologiques des personnes jouent un rôle important dans la prise de décision concernant le départ. La capacité des parents et proches déjà installés en Turquie de recevoir et de prendre un certain temps en charge le reste de la famille est un facteur important. Même si pendant un certain temps les autorités turques demandent un visa aux ressortissants de Bulgarie, on ne leur crée pas beaucoup de difficultés. Au consulat, certains donnent des dessous de table pour obtenir le visa. L'émigration clandestine est une autre option. Nombreuses sont les histoires de traversée de la frontière avec un visa "aller simple» ou dans l'arrière de bus ou de trains avec l'aide d'un réseau de trafic de personnes?.

Dans la période de 1989 à 2001, les autorités turques attribuent la nationalité «à titre exceptionnel » à environ 16000 personnes ${ }^{10}$. Or, selon les données non officielles, les immigrants de Bulgarie sont beaucoup plus nombreux ${ }^{11}$. Selon les experts, dans la période de 1989 à 1996, les nouveaux arrivants de Bulgarie comptent environ 400000 
personnes ${ }^{12}$. Leur nombre augmente à partir de 2000 avec la suppression du régime des visas. A partir de cette époque on peut passer librement la frontière avec un visa de séjour de trois mois. Le réseau de transports transfrontaliers qui se développe de plus en plus facilite la circulation des personnes entre les deux pays.

Dans les dernières années, on observe une nouvelle vague de migration de la Bulgarie vers la Turquie. Nous pourrions l'appeler « la migration des brues ». Il s'agit de femmes turques de Bulgarie qui se marient à des immigrants déjà établis en Turquie ou à leurs enfants. De fait, la minorité turque de Bulgarie est également touchée par le phénomène d'effondrement démographique et le vieillissement de la population caractéristiques de l'ensemble de la société bulgare. La décision de partir se marier en Turquie repose sur deux facteurs essentiels : d'une part, les jeunes femmes célibataires ne trouvent pas de partenaire en Bulgarie, d'autre part, dans les représentations collectives, la Turquie est perçue comme offrant de meilleures conditions de vie.

7 Parmi ces jeunes femmes beaucoup ont fait partie de la vague de retours en Bulgarie de l'automne 1989-l'hiver 1990 après la «Grande excursion ». La circulation transfrontalière de plus en plus libre, le tourisme, les différentes fêtes familiales et religieuses facilitent les rencontres entre femmes turques en visite et immigrants turcs installés en Turquie. Les rencontres se font également en Bulgarie, lorsque les émigrants sont de retour pour quelque occasion ou dans le milieux étudiants lorsque les jeunes femmes de Bulgarie partent en Turquie faire des études supérieures. On observe que les jeunes couples mariés préfèrent s'établir définitivement en Turquie où ils estiment que les conditions de vie et les salaires sont meilleurs qu'en Bulgarie. De même, si l'on compare les immigrants originaires de Bulgarie à d'autres groupes de migrants on remarque que les Turcs bulgares viennent pour s'établir de manière permanente en Turquie et non pour transiter vers un pays d'Europe occidentale ou vers les Etats-Unis ${ }^{13}$.

Lors de la "Grande excursion » de l'été 1989, les candidats à l'immigration traversent la frontière bulgaro-turque avec très peu d'affaires personnelles: "Seulement des vêtements, couvertures, couettes, des choses en textile. Je ne pouvait pas transporter en Turquie mes meubles de cuisine par exemple.... ${ }^{14}$. Ensuite, ils sont installés dans des écoles désertes en été ou dans des campements avec des tentes construits spécialement pour eux. A l'automne avec le début de l'année scolaire, les familles sont transférées dans camps de vacances, propriété de l'Etat. D'autres camps de réfugiés ont été construits les villes de Edirne, Istanbul, Bursa, Izmir, Tekirdag, Eski Shehir, Ankara, Bolu, etc ${ }^{15}$. Le gouvernement prévoyait au début d'installer les réfugiés dans la partie orientale de l'Anatolie: « Nous les Göçmeni (les déplacés) on est comme des soldats. Tu vas là où on te dit. $\rrbracket^{16}$ Cette région est peu peuplée et à majorité kurde. Les personnes qui y vont, restent peu de temps et partent ensuite vers les parties occidentales de la Turquie. Pour la plupart, ils disent ne pas pouvoir s'adapter au climat rude de certaines régions et préfèrent des régions " plus civilisées » où ils peuvent trouver du travail. En conséquence de ces migrations internes, effectuées dès le premiers mois et à l'initiative des migrants, environ 100000 personnes échappent au contrôle des autorités turques. Une autre partie des nouveaux arrivants se dirige vers les grandes villes comme Bursa (80 000 réfugiés) ou encore Istanbul et Edirne. A Bursa par exemple, le flux massif d'immigrants qui arrivent par vagues dans la ville oblige les autorités locales à construire des barrières autour de la ville, incapable d'accueillir plus de réfugiés ${ }^{17}$. 
Une partie des immigrants rejoignent des parents qui ont quitté la Bulgarie lors de vagues d'émigration précédentes (1950-51 et 1968-78). Les déplacements sont effectués avec l'assistance des autorités locales et de l'Etat : «Là-bas il y avait des agents publics qui nous prenaient en charge. Ils nous conduisaient là où on avait des parents proches. Non pas avec des bus mais en voiture $\aleph^{18}$. Le séjour chez les parents est bref, pas plus de quelques jours selon les interrogés. La raison principale en est le manque d'hospitalité des proches qui ne les "supportent pas plus de deux-trois jours». D'autres, qui ne peuvent pas compter sur l'aide de la famille sont pris en charge par des institutions religieuses musulmanes et certains, sans logement ni travail, sont même hébergés dans des mosquées. «Au début, mon père ne pouvait pas trouver du travail et nous avons passé un an dans une pièce de la mosquée locale, prévue pour héberger des gens pauvres. $»^{19}$ Bien qu'ils soient originaires de zones rurales en Bulgarie, les migrants préfèrent s'établir à proximité ou dans les villes. "On s'y est fait difficilement. Nous habitions de petites villes et nous voulions vivre dans une petite ville ici aussi mais il n'y a pas de travail donc nous sommes venus vivre en ville. ${ }^{20}$

10 Les populations immigrées en Turquie se rendent au début à Edirne et à Istanbul, dans la partie européenne. Dans un second temps, elles se déplacent vers d'autres villes du pays et, pour ce qui est des Turcs de Bulgarie, ils préfèrent les villes de Bursa, Izmir, Çorluet Karadag. Plusieurs facteurs essentiels sont intervenus dans le choix de la ville d'établissement. Premièrement, il est fonction des vagues d'immigration précédentes, pas seulement de Bulgarie mais aussi d'autres pays des Balkans et de l'Asie. Les immigrants bulgares préfèrent rejoindre les colonies d'immigrants déjà constituées dans les grandes villes turques ou devenues elles-mêmes des bidonvilles: «J'ai vécu 4 ans à Istanbul, je n'ai pas pu m'y faire du tout. Trop de gens ce n'est pas pour nous. A Çorlu ça va, c'est une ville d'immigrants, il y a $80 \%$ des gens qui viennent de la Bulgarie, de la Yougoslavie, de la Grèce, de la Thrace occidentale. $»^{21}$

11 Un autre facteur qui intervient dans le choix concerne les conditions climatiques. Certains se rendent à Bursa parce que le climat est semblable à celui de la Bulgarie, d'autres préfèrent aller dans le sud à Izmir ou au bord de la mer Egée parce qu'il y fait plus chaud en hiver. Le marché du travail constitue un autre facteur important. «J'ai choisi Izmir parce que je me suis dis c'est une grande ville, il y a un port, un aéroport, ça veut dire qu'il y a des perspectives $»^{22}$. Ils préfèrent les grandes villes parce que « làbas c'est différent, il y beaucoup de fabriques de textiles entre Lüleburgaz et Çorlu alors qu'il n'y en a pas à Istanbul. Dans ces fabriques il n'y a pas seulement nous, il y a aussi des ouvriers de l'Anatolie, pour la plupart des Kurdes $»^{23}$. Les individus affichent différentes préférences selon les classes d'âge. Par exemple les plus âgés se sont installés à Bursa, ville "plus religieuse » et les plus jeunes vont à Izmir, qu'ils estiment plus moderne et européenne. En effet, les jeunes après un certain temps passé avec leurs parents se sont déplacés vers le sud, la partie européenne de la Turquie dite « la Thrace » ou la Turquie occidentale avec sa côte égéenne.

12 Pour les nouveaux arrivants le critère de "l'européanité » de la ville est important. Ainsi, dans une ville " plus européenne » ils ne vont pas se sentir gênés de porter « des vêtements européens ", les femmes peuvent porter tranquillement des jupes et des pantalons. «A Izmir les femmes ne sont pas voilées, les gens sont plus ouverts, comme en Bulgarie. On peut porter des shorts, des pantalons, aller partout, comme dans une ville européenne. A Ankara, à Bursa ce n'est pas comme ça. Certaines personnes vont 
peut-être faire pression pour que tu t'adaptes à eux, ils vont te regarder d'un autre œil. Si je n'avais pas pu venir à Izmir, je serais retourné en Bulgarie. Honnêtement ${ }^{24}$.

13 La confession est un autre critère important. Les Turcs de Bulgarie de confession alevi ${ }^{25}$ se dirigent vers Istanbul et Çorlu. Même leurs connaissances littéraires interviennent dans le choix et la représentation du lieu, comme c'est le cas pour Izmir, selon un informateur : « Nous avons connu Smyrne par les écrits de Hristo Smirnenski ${ }^{26}$ ».

14 A la suite de leur immigration en Turquie, les Turcs de Bulgarie doivent s'adapter aux nouvelles conditions politiques, économiques et sociales. On observe trois stratégies d'adaptation : l'acculturation et l'intégration ; l'opposition ou la réaction; le retrait et la marginalisation. Si les nouveaux arrivants conservent des éléments de leur culture d'origine, ils empruntent aussi à la culture d'accueil. Les relations d'interaction avec la société locale sont dynamiques et varient sur une échelle qui va de l'intercompréhension au conflit déclaré. Dans ce cas les migrants maintiennent les relations avec leur pays d'origine, ce qui conduit à la construction d'une communauté transnationale ${ }^{27}$. Plusieurs étapes peuvent être distinguées dans le processus d'adaptation. Premièrement, le groupe cherche à démarquer son territoire, les acteurs se l'approprient progressivement comme étant celui de leur espace de vie. Avec le temps cette espace vient à être pensé comme sien. Dans ce processus le degré de concentration ou de dispersion du groupe sur le territoire est important. La deuxième étape dans l'appropriation du nouvel espace débute avec la construction d'un logement familial. On considère que l'acte de vendre sa propriété dans le pays d'origine suivi de l'achat d'une nouvelle dans le pays d'accueil montre le degré d'avancement du processus d'intégration. La troisième étape concerne la construction des topos ou lieux de rassemblement entre immigrants tels que le marché, le restaurant, le centre culturel où l'on se rencontre régulièrement. A travers ces différentes étapes les immigrants tentent d'organiser le nouvel espace de vie et créent une culture subjective, afin d'obtenir le droit d'exercer un contrôle sur leur territoire ${ }^{28}$. Le statut socioprofessionnel, le niveau d'éducation et la participation des migrants à la vie économique locale jouent un rôle essentiel dans le processus d'insertion et dans la construction des hiérarchies sociales. L'obtention de la nationalité turque représente une étape importante.

Les réseaux informels sont de même essentiels à la survie des groupes de migrants. Ceux-ci comprennent les relations d'entraide entre amis, parents, voisins, individus dont l'ensemble assure le processus d'adaptation et d'insertion du groupe. Ces réseaux relient les migrants à leur groupe d'origine, dans le lieu d'origine mais aussi avec les autres membres de la communauté vivant dans d'autres régions de Turquie. La mise en place d'organisations et d'associations qui non seulement défendent les droits des immigrants mais aussi servent de relais entre différentes communautés représente un stade supérieur dans l'usage de ces réseaux. Les activités en commun nourrissent les relations entre les individus et renforcent le sentiment d'appartenance au même groupe.

16 Pour les individus, l'exode de l'été 1989 a représenté un bouleversement radical dans leur existence, amenant de nouvelles épreuves et difficultés à survivre dans le nouveau contexte. Pour ces hommes et femmes il s'agit de "recommencer sa vie à zéro ". Souvent les Turcs bulgares opposent d'un coté la « belle vie » dans le passé, en Bulgarie, à celle d'aujourd'hui : "Là-bas c'était la vie, alors qu'ici on rame et on s'accroche pour survivre $\aleph^{29}$. Selon les personnes interrogées, les premières années sont les plus 
difficiles; pour survivre, il faut s'adapter, faire attention, mesurer ses actes, ne faire confiance à personne. «L'immigré doit avoir des yeux dans le dos " $^{30}$.

17 La plupart des immigrés Turcs de Bulgarie se sentent rejetés dans leur pays d'origine, sans être pour autant acceptés dans la nouvelle patrie. Ainsi se crée le sentiment d'appartenir à une minorité en Turquie même. Il existe différentes catégories de migrants selon les parcours, mais aussi selon l'identité ethnique et culturelle qu'ils privilégient. Ainsi, on peut distinguer trois groupes : les migrants (ceux qui restent en Turquie); les ré-émigrants (ceux qui, déçus de la nouvelle patrie, reviennent en Bulgarie) ; les personnes naturalisées.

\section{II - Autodéfinitions identitaires et expériences vécues en Turquie}

$18 \mathrm{Au}$ XIXème siècle, dans l'Empire ottoman multiethnique, la population turque dans la région était considérée comme majoritaire et l'élite turque était dominante. Après la dissolution de l'Empire, une partie importante des sujets turcs et musulmans s'est trouvée incluse dans les territoires des nouveaux Etat-nations balkaniques. Dans l'Etat bulgare, la communauté turque a subi un processus d'acculturation qui a conduit à la différencier de plus en plus des Turcs de la "mère-patrie », la Turquie. Devenus une minorité, les Turcs de Bulgarie ont développé un sentiment d'appartenance ethnique et le groupe, conscient de sa différence, s'est davantage refermé sur lui-même. La politique de l'Etat bulgare a également contribué à la construction des différences et le groupe ethnique turc à pu conserver certains traits culturels comme la langue et la religion. Si la communauté a pu se préserver, c'est aussi en réaction à la violente politique d'assimilation entreprise par les autorités bulgares dans certaines périodes de l'histoire. Dans la formation d'un sentiment d'appartenance ethnique, la politique turque a également joué un rôle : elle a défendu les droits de « sa » minorité en Bulgarie mais au-delà, ses intérêts politiques et économiques dans la région.

19 Après l'émigration en Turquie, le sentiment de différence avec les Turcs de Turquie apparaît de plus en plus structurant pour les migrants. Cette différence explique le vaet-vient constant entre les deux "patries", mobilité qui construit une identité de migrant $^{31}$. Un des phénomènes propres au monde moderne et globalisé concerne la disparition des frontières, la circulation intense des individus et des groupes et ses effets induits sur leurs représentations et leur identifications. Tel est le cas également pour les Turcs de Bulgarie aux identités multiples et changeantes. Les identifications des migrants varient en fonction des situations et des individus. C'est pourquoi il semble difficile de les catégoriser en fonction de marqueurs tels que l'ethnicité, la religion, la langue ou la culture.

Les communautés de migrants sont des «communautés transnationales». Elles dépendent à la fois de la politique de l'Etat de départ et de l'Etat d'accueil. La communauté de migrants en tant que telle dépend de la relation à ces deux Etats et les individus sans cesse circulent, dans le double objectif de conserver leur model culturel d'avant et d'en acquérir un autre qui leur permettra d'accéder à une meilleur statut socio-économique. Les caractéristiques principales de ce groupe sont: la double nationalité, la mobilité constante, un peuplement compact, des activités en commun, la diglossie, le bilinguisme, la conservation des pratiques religieuses et des traditions 
culturelles, l'endogamie et enfin un discours de valorisation de ces marqueurs. Dans le comportement des migrants au quotidien, toutes ces valeurs peuvent être observées :

\begin{tabular}{|c|c|}
\hline Double nationalité & $\begin{array}{l}\text { On conserve ou on recouvre la nationalité de l'Etat d'origine. } \\
\text { On acquiert la nationalité de l'Etat d'accueil }\end{array}$ \\
\hline Mobilité intense & $\begin{array}{l}\text { Mobilité intense entre l'Etat d'origine, l'Etat d'accueil et d'autres } \\
\text { pays. }\end{array}$ \\
\hline $\begin{array}{l}\text { Peuplement compact dans les } \\
\text { quartiers/villes/villages }\end{array}$ & $\begin{array}{l}\text { Construction de quartiers nouveaux et des topos demigrants (lieux } \\
\text { de culte, marchés, magasins, cafés, restaurants, etc.) }\end{array}$ \\
\hline Activités en commun & Entraide, rencontres villageoises, célébrations de fêtes... \\
\hline Diglossie & Maîtrise de différentes langues orales et/ou standardisées \\
\hline Bilinguisme & $\begin{array}{l}\text { Maîtrise orale de la langue officielle du pays d'origine et du pays de } \\
\text { destination }\end{array}$ \\
\hline $\begin{array}{l}\text { Conservation des pratiques } \\
\text { religieuses }\end{array}$ & $\begin{array}{l}\text { Maintien de la variante locale de la religion pratiquée dans le pays } \\
\text { d'origine }\end{array}$ \\
\hline $\begin{array}{l}\text { Conservation de la culture } \\
\text { traditionnelle }\end{array}$ & Perpétuation des pratiques rituelles et festives d'origine \\
\hline Endogamie & Mariage à l'intérieur du groupe de migrants \\
\hline Valorisation de la culture & $\begin{array}{l}\text { Les acteurs se représentent leur culture comme étant la plus } \\
\text { «pure » et la «vraie». }\end{array}$ \\
\hline
\end{tabular}

Source : tableau réalisé par l'auteur

21 La double nationalité est un marqueur particulièrement important ${ }^{32}$. Pour les Turcs de Bulgarie, les deux nationalités désignent à la fois leur identité nationale et le lien originel avec la société dans laquelle ils ont été élevés. Cette double citoyenneté permet à cette communauté transnationale de participer à la vie politique en exerçant son droit de vote à la fois dans le pays d'origine et dans le pays d'accueil. La mobilité intense est une des caractéristiques essentielles du monde globalisé et de la société post-moderne. Les Turcs de Bulgarie circulent ainsi non seulement entre la Bulgarie et la Turquie, mais aussi en Europe occidentale et aux Etats-Unis pour des raisons telles que le travail, les vacances, les biens à gérer, des parents à visiter, l'accès aux soins médicaux ou encore les fêtes.

Les migrants avec lesquels des entretiens ont été réalisés lors des missions de terrain de 2005 ont des représentations ambivalentes de leurs deux patries. L'Etat d'origine revêt pour eux plusieurs visages aux traits à la fois positifs et négatifs. Les personnes interrogées parlent de "patrie d'origine " où l'on a vécu son enfance, sa jeunesse, où ont été abandonnés les propriétés et les biens de famille. La terre d'origine est qualifiée de "jardin d'Eden », celle qui appartiendra bientôt à l'Union européenne (UE) ${ }^{33}$. Mais aussi c'est aussi un lieu de discrimination et de crise économique. La Bulgarie est 
présente dans les souvenirs du passé, mais elle apporte aussi des avantages au présent: des papiers d'identité bulgares et donc européens, qui permettent de voyager sans visa à destination de certains pays. En Bulgarie, les migrants soulignent qu'ils ont pu faire de bonnes études et encore aujourd'hui, grâce à la double nationalité, ils peuvent bénéficier du système scolaire et de la sécurité sociale bulgares, par exemple. Ce n'est pas un hasard sur de nombreux Turcs installés en Turquie préfèrent voir leur enfant accomplir leurs études supérieures en Bulgarie, particulièrement depuis que le pays est membre de l'Union européenne. Evidemment, cette question présente aussi un aspect financier dans la mesure où l'enseignement supérieur est moins onéreux en Bulgarie qu'en Turquie, d'autant plus que la détention de la citoyenneté bulgare permet d'obtenir des avantages connus en la matière.

Aux yeux de la plupart des migrants, le pays d'accueil représente la patrie historique d'où sont venus leurs ancêtres, il y a des siècles. Aujourd'hui, la Turquie accueille de nouveau ses enfants et les aide à s'épanouir dans la vie; elle leur permet de vivre librement leur identité de Turcs et de musulmans. Avec le temps, les migrants réalisent que la nouvelle patrie n'est pas celle des rêves, des mythes et des légendes dont ils ont entendu parler depuis des générations. Elle leur est apparue comme différente et inconnue, leur offrant une vie difficile et pleine de péripéties. Cependant, au long du processus d'adaptation, les immigrants la découvrent et modifient leurs représentations afin de s'approprier leur nouvelle patrie et de la concevoir comme étant un véritable « chez soi ».

La concentration et la densité du peuplement d'immigrants par lieu d'habitation constitue un autre critère essentiel à la conservation et au renouvellement des «traditions anciennes» du groupe. C'est pourquoi les nouveaux arrivants sont à l'origine de nouveaux espaces urbains et créent de nouveaux quartiers, comme par exemple celui de Cörece, près d'Izmir. Certains ont été construits par l'Etat précisément pour accueillir les Turcs de Bulgarie. Ils sont situés dans les environs des grandes villes et représentent d'immenses complexes urbains où s'élèvent des blocs en béton. Les appartements sont attribués par loterie, mais la priorité est accordée aux victimes de la répression durant la politique d'assimilation en Bulgarie dans les années 1980. Les Turcs bulgares qui vivent à Izmir et à Istanbul ont préféré s'établir dans des villages proches des centres urbains et y construire leur quartier d'habitation. L'Etat turc les autorise à acheter des terrains à des tarifs préférentiels et à y construire leurs maisons. A Izmir, ce sont les quartiers de Sarnach et de Buca. Les migrants des différentes régions bulgares non seulement souhaitent s'établir en un même lieu avec tous leurs proches, mais ils cherchent aussi à échapper aux mégapoles turques. Telle est la raison pour laquelle ils préfèrent vivre dans les quartiers périphériques des grandes villes, qui ressemblent davantage aux petits villages qu'ils ont quittés. Dans le même temps, le choix d'une migration en zone urbaine s'impose pour des raisons pratiques: les précédentes vagues migratoires ont été également dirigées vers les villes. En outre, les grandes mégapoles donnent plus de possibilité de réalisation professionnelle et d'accès à l'éducation. Dans le même temps, les migrants s'emploient à changer radicalement leur vie et à recommencer à zéro.

Les Turcs de Bulgarie construisent des maisons à deux ou à trois étages, d'un style caractéristique dans tout le pays. Ils assurent eux-mêmes la construction des maisons selon un principe d'entre-aide, contribuant au renforcement des solidarités et du sentiment d'intégration dans la société turque. Lorsqu'ils expliquent leur 
empressement à construire des maisons, les personnes interrogées se réfèrent aux " habitudes de Bulgarie ", au désir d'avoir "sa » maison et au modèle patrilocal de leur société d'origine. Ainsi, ils ont le sentiment de "faire pousser des racines" dans la nouvelle patrie ${ }^{34}$, ce qui équivaut à un établissement définitif. La nouvelle maison incarne la fin de l'espoir de revenir dans l'ancienne patrie et le début d'une nouvelle vie dans une nouvelle patrie. Cependant, peu de migrants se décident à vendre leurs propriétés en Bulgarie et ils préfèrent les louer à des parents ou même laisser en jachère leurs terres cultivables. Ces choix prouvent que leur ré-enracinement dans la nouvelle patrie ne met pas radicalement fin aux liens avec la Bulgarie et que les individus ne peuvent pas véritablement rompre avec leur passé.

Le développement de nouveaux quartiers comprend aussi la construction de mosquées et de marchés, ces derniers ayant la particularité de représenter la culture culinaire des migrants et de les réunir les jours de marché. Le marché est ouvert un jour par semaine ou bien tous les jours, comme à Buca. Sa principale fonction est de fournir les habitants en produits en provenance de Bulgarie: ces produits sont apportés par bus, sur des lignes régulières quotidiennes entre la Bulgarie et la Turquie. Il est fréquent que le différentiel de prix soit inexistant, mais les migrants installés en Turquie les considèrent comme plus "savoureux ». Les immigrants se disent "habitués " à consommer des produits bulgares, qui sont de "très bonne qualité ». Les habitants importent également des habitudes ou des activités pratiquées pendant leur temps libre en Bulgarie. Ainsi les hommes vont-ils à la chasse au gibier ${ }^{35}$, alors que les femmes aiment se réunir au café du quartier. Le processus d'acculturation ne détruit pas le modèle culturel spécifique.

Les activités en commun contribuent au renforcement des solidarités au sein du groupe et démontrent un certain degré de fermeture. Les individus partagent la même histoire et les mêmes problèmes aujourd'hui, ils rencontrent compréhension et soutien seulement au sein du même groupe. Ils s'aident mutuellement pour résoudre des difficultés personnelles, trouver du travail, construire une maison. Ils sont ensemble dans leurs activités économiques mais aussi lors des fêtes de famille, des fêtes religieuses. Ils organisent souvent des rencontres villageoises, pour se parler et se souvenir «du bon vieux temps» en Bulgarie, autour de repas et de verres de rakia importés depuis là-bas. Ainsi se construit un sentiment d'appartenance à une identité locale et régionale particulière qui, au-delà du village ou de la ville d'origine, rassemble les individus en immigration par le partage de traits culturels communs : on parle ainsi des « gens » du Džebel, de Cernoocen, de Kărdžali, etc.

A la suite de leur arrivée en Turquie, les Turcs de Bulgarie ont constaté des différences importantes entre leur langue turque et celle des populations locales. Ils disent qu'ils parlent « une autre langue » qui présente des caractéristiques empruntés au vieux turc ottoman, plus exactement au dialecte oguz ${ }^{36}$. Cette forme archaïque de la langue orale turque a pu être conservée en Bulgarie ${ }^{37}$ et aujourd'hui elle est toujours pratiquée parmi les migrants en Turquie, même lorsque ces derniers ont appris la langue turque standardisée. Au sein du groupe ils continuent à communiquer dans leur parler d'origine. De même, dans leurs communications privées, ils emploient la langue bulgare. Ainsi la diglossie et le bilinguisme construisent et renforcent le sentiment d'être différent des autres.

L'appartenance religieuse est un autre marqueur important dans la construction des identifications sociales et culturelles. Dans la patrie de tous les Turcs, les Turcs de 
Bulgarie découvrent, déçus, qu'ils pratiquent une variante locale de l'islam assez différente de celle pratiquée en Turquie. Si cette prise de conscience représente, pour les plus âgés, une véritable tragédie, les jeunes et moins jeunes n'en font pas grand cas. En effet, les personnes âgées sont réellement pratiquantes, alors que les jeunes semblent avoir principalement recours à la foi en situation de crise. De fait, le marqueur de la pratique religieuse contribue également à renforcer le sentiment de différence entre le groupe immigrant et la société d'accueil. Il en est de même pour les rites et les coutumes représentant des variantes locales en Bulgarie. En Turquie les éléments qui différencient la culture traditionnelle locale de celle des migrants sont perçus comme une frontière démarquant les locaux des nouveaux arrivants. Les Turcs bulgares considèrent certaines pratiques issues de leur tradition comme très conservatives. Ils donnent l'exemple du rituel de consommation de nourriture et de la danse accompagnant les rites de passage tels que le sunet (la circoncision) et le mariage. La perpétuation de ces pratiques en Turquie permet d'assurer non seulement la socialisation religieuse des jeunes mais aussi leur socialisation au sein du groupe d'immigrants, en tant que Turcs de Bulgarie. Les spécificités linguistiques, la religion, les pratiques festives et cultuelles particulières expliquent l'endogamie au sein du groupe des migrants de Bulgarie. Cela conduit au cloisonnement de la communauté et à la conservation du modèle culturel ancien.

Les Turcs de Bulgarie se conçoivent comme supérieurs aux Turcs de Turquie, ils estiment avoir conservé l'identité ethnique, la langue, la foi et les traditions « les plus pures ». La population d'origine locale ne les considère pas comme étant " des nôtres » non plus. Elle voit en eux l'Autre ou l'étranger, des porteurs des marques de l'identité bulgare et de la chrétienté. Les comportements sociaux, les attitudes corporelles et gestuelles, les vêtements, les normes morales que les nouveaux arrivants apportent avec eux sont perçus comme les marques de cette culture étrangère à la Turquie. De même, aux yeux des locaux ils représentent une menace pour l'emploi, ils " profitent " de la sécurité sociale et des aides de l'Etat et lorsqu'ils réussissent socialement, leur ascension sociales est également mal perçue. Le rejet de la population locale vis-à-vis des immigrants de Bulgarie apparaît dans les dénominations collectives : les Turcs de Turquie les appellent «Gjauri » (incroyants) ou « Bulgarlar », exonymes qui stigmatisent en attribuant une identité ni turque, ni musulmane. En définitive, l'identité collective des migrants oscille entre celle de l'Etat d'origine et de l'Etat d'accueil, ce qui précisément fait du groupe une communauté transnationale.

\section{III - Les trajectoires des « ré-emigrants » et des « naturalisés »}

Parmi les immigrants de Bulgarie en Turquie, certains n'ont pas réussi à trouver leur place dans la société d'accueil ; entre 1989 et 1990, plus de 152000 personnes sont ainsi rentrées en Bulgarie. Un informateur relate son expérience en ces termes : «Nous, on avait des parents là-bas, mais ils ne nous ont pas aidés. Finalement, ceux qui sont allés dans les camps pour immigrés s'en sont sortis mieux que nous car l'Etat leur a donné des maisons, alors que nous n'avons rien eu, ni logement, ni travail " ${ }^{38}$. D'autres rentrent parce qu'ils ont en Bulgarie des parents âgés qui ont refusé de partir, mais dont ils doivent s'occuper. D'autres encore n'acceptent pas la nouvelle réalité économique, sociale et culturelle de Turquie et ne supportent pas l'attitude méprisante et le rejet des Turcs locaux. Ainsi le 
refus ou l'incapacité de s'adapter au nouveau contexte les conduit-il à migrer de nouveau et massivement vers la Bulgarie, dès avant la chute du communisme en novembre 1989, mais également dans les premiers mois du nouveau régime. Même si leur identité ethnique et religieuse coïncide avec celle du pays d'immigration, pour eux, l'identification à la Bulgarie comme pays d'origine prédomine. L'identité bulgare prend le dessus sur les identifications ethniques et religieuses.

La catégorie des personnes "naturalisées » inclut les migrants qui réussissent à s'intégrer totalement dans le pays d'accueil. Dans le processus d'acculturation ils parviennent à « oublier » qu'ils ont été des immigrés. Généralement il s'agit d'individus dispersés sur le territoire de la Turquie, vivant dans les quartiers anciens avec les locaux qui n'entretiennent pas de relations régulières avec les autres immigrants de Bulgarie. Leur relation au pays d'origine est presque interrompue, ils n'ont pas de parents là-bas, ni de biens immobiliers. Ils se marient avec des Turcs de Turquie et ne parlent plus que la langue littéraire turque (et non des dialectes locaux et régionaux, dans la mesure où ils espèrent être ainsi mieux acceptés). Leur identité ethnique coïncide avec leur identité nationale. Ils ont délaissé leurs traditions d'origine et pratiquent les rites et les coutumes du pays d'accueil ${ }^{39}$.

\section{Conclusion}

On peut difficilement prévoir les processus identitaires que les Turcs de Bulgarie connaîtront à l'avenir. Leur future trajectoire est susceptible de dépendre de nombreux facteurs: la situation économique et politique en Turquie comme en Bulgarie, les politiques des Etats à leur égard. L'admission de la Bulgarie au sein de l'Union européenne a eu un impact dont il est sans doute trop tôt pour prendre pleinement la mesure ; certains migrants envisageaient avant l'adhésion de rentrer en Bulgarie. On peut également supposer que de nombreux individus d'âge moyen rentreront en Bulgarie après la retraite. Certains indicateurs témoignent de ces intentions comme les vagues d'acquisition de propriétés dans les zones mixtes (Turcs et Bulgares), qui ont entraîné une forte hausse des prix de l'immobilier dans ces régions. Une partie des immigrants va certainement transiter par la Bulgarie dans l'objectif de s'établir en Europe de l'Ouest ou aux Etats-Unis. Pour ceux qui resteront en Turquie, le processus d'assimilation s'achèvera avec succès, comme cela était le cas pour les générations précédentes. On peut émettre l'hypothèse que les particularismes culturels du groupe migrant disparaitront progressivement ainsi que le sentiment d'appartenance des individus à une identité collective à part, celle des Turcs de Bulgarie.

\section{NOTES}

1. Selon les données du dernier recensement en 2001, se sont déclarés Turcs environ 746664 personnes, soit $9,41 \%$ de la population totale. 
2. Cet article se base sur des données recueillies lors d'enquêtes de terrain effectuées en Bulgarie, parmi des Turcs de retour dans leur pays d'origine pour une courte période (1999-2005). De même, entre mars et septembre 2002, j'ai conduit une enquête dans les quartiers de Buca, Bornova, Görece et Sarnic construits aux environs de villages non loin d'Izmir, en Turquie. La plupart des immigrés Turcs bulgares sont originaires de la région de Haskovo et de Kardzhali en Bulgarie du sud. Les entretiens - environ 140 au total, en Bulgarie et en Turquie - ont été menés en bulgare et/ou en turc, en fonction des interlocuteurs, de l'environnement et du contexte de l'étude. L'auteur a choisi la langue de discussion en fonction de chaque situation spécifique.

3. Voir sur cette question : Eroğlu (Hamza), « The Question of Turkish Minority in Bulgaria from Perspective of International Law », in The Turkish Presence in Bulgaria. Ankara, Türk Tarih Kurumu Basımevi, 1986, pp. 59-90; Memişoğlu (Hüseyin), TheBulgarian Oppression in Historical Perspective, Ankara, Devran Matbaasi Necatibey Cad, 1989; şimşir (Bilal), Contribution a l'histoire des populations turques en Bulgarie (1876-1880), Ankara, TKA, 1966.

4. Zhivkov (Todor), « Etnokulturno razvitie na Vuzroditelnia protses », in Problemi na razvitieto na balgarskata narodnost i natsia, Sofia, BAN, 1988, pp. 127-143; Zhivkov (Todor), Memoari, Sofia, Siv, AD, 1997; Zagorov (Orlin), Vuzroditelniyat protses. Teza. Antiteza. (Otritsanie na otritsanieto), Sofia: Pandora, 1993. Mihailov (St),Vuzrozhdenskiyat protses $v$ Bulgaria,Sofia, M\&M, 1992; Tahirov (Sh), Edinenieto, Sofia, OF, 1981.

5. Plusieurs sources statistiques différentes proposent des évaluations contrastées du nombre des Turcs ayant quitté le pays. L'auteur a donc retenu un chiffre moyen.

6. Karamihova (Margarita), « T. nar. "Vuzroditelen protses”. Politika i rezultati 1987-1997 ». In Za promenite (sbornik), Sofia, 1997, pp. 397-398.

7. Zhelyazkova (Antonina), «Turcite v Bulgaria " in Dve, Vera Moutafchieva and Antonina Zhelyazkova, Sofia, 2002, pp. 161-197.

8. Voire les archives de l'Institut d'Ethnographie et Musée, Sofia : (AEIM), № 574-III, 27.

9. Le nombre d'immigrés clandestins arrêtés en provenance de Bulgarie pour la période entre 1994 et juin 2004 s'élève à 9111 personnes. Voir : Apap (Johanna), Carrera (Sergio) and Kirişci (Kemal). "Turkey in the European Area of Freedom, Security and Justice», EU-Turkey Working Paper, $\mathrm{n}^{\circ} 3$, Brussels: Centre for European Policy Studies, 2004, p. 18. Voir aussi : BaldwinEdwards (Martin). "Migration in the Middle East and Mediterranean. A Regional Study prepared for the Global Commission on International Migration ", January 2005, p. 13 (http:// www.gcim.org/attachements/RS5.pdf).

10. Stojanov (Valeri). « Turskoto naselenie v Bulgaria mejdu polusite na etnicheskata politika », Sofia, 1998, pp.

11. Selon les informations de l'Agence de Presse Bulgare (BTA) du 12 février 2001, les ressortissants bulgares d'origine turque ont lancé un appel au ministre de l'Intérieur afin d'accélérer le processus de régularisation de leur situation et celui de l'attribution de la nationalité turque à ceux qui sont venus après 1992. Selon les auteurs de l'appel : «Les Turcs bulgares qui n'ont pas été naturalisés se trouvent obligés chaque année de payer environ 100 dollars de frais pour l'obtention de la carte de séjour. Cela crée de grandes difficultés financières pour des familles généralement nombreuses et à bas revenus. Pour ceux qui sont venus de Bulgarie avec des visas de touristes ou clandestinement il y a un autre problème : ils sont traités comme des clandestins et ils n'ont d'autre choix que de se cacher, sans papiers, ni assurance maladie. »

12. Zhelyazkova (Antonina), «Turcite v Bulgaria », op. cit. p. 192.

13. Icduygu, (A.), «Irregular Migration in Turkey »- IOM Migration Research Series, 2003, № 12.

14. AEIM, № 574-III, 55.

15. Dimitrova (D), « Balgarskite turci precelnitsi v Republika Turcia prez 1989 godina. Adaptacia i promeni v kulturnia model ». In Zhelyazkova (Antonina), dir.,Mezdu adaptaciata $i$ nostalgiata. Balgarskite turci $v$ Turcia, Sofia, MCPMKV, 1998, pp. 76-139, op. cit. p. 84. 
16. Dimitrova (D), op. cit, p. 85.

17. Ibidem, p. 84.

18. Arhiv na Megdunarodnia centar za izsledvane na malcinstvata $i$ kulturnite vzaimodeistvia (AMCIMKV) N 3321/19. 02.2004, p. 188.

19. http//www.orhanco.com

20. AEIM, N574-III, p. 18.

21. AMCIMKV, Nº3321/19. 02.2004, p. 224.

22. AEIM, N574-III, 13.

23. AMCIMKV, $\mathrm{N}^{\circ} 3321 / 19.02 .2004$ г., 201

24. AEIM, $\mathrm{N}^{\circ} 574-\mathrm{III}, 55$

25. Sur les musulmans hétérodoxes en Bulgarie, voir Georgieva (Ivanichka) (ed.). « Bulgarskite aliyani » [Les Alijani bulgares], Sofia : Universitetsko izdatelstvo, 1991; Gramatikova (Nevena). « Isljamski neortodoksalni techeniya $\mathrm{v}$ bulgarskite zemi »[Les courants musulmans non orthodoxes sur les terres bulgares], In Gradeva (Rositsa), Istoria na mjusjulmanskata kultura po bulgarskite zemi [Histoire de la culture musulmane sur les terres bulgares], Sofia, 2001, pp. 192-284 ; Karamihova (Margarita). «Prikazka za Osman baba » [Récit sur Osman baba], Sofia, 2002; Mikov (Ljumobir) « Izkustvoto na heterodoksnite mjusjulmani v Bulgaria (XVI-XX vek). Bektashi i alevi/alevi »[L'art des musulmans hétérodoxes en Bulgarie (XVIè-XXè siècle). Bektaschi et alevi/alevi]. Sofia, Akademichno izdatelstvo "Marin Drinov", 2005; Aleksiev (Bojidar), «Folklorni profili na mjusjulmanskite svetsi v Bulgaria »[Profils folkloriques des saints musulmans en Bulgarie], Sofia, Akademichno izdatelstvo "Marin Drinov", 2005.

26. Hristo Dimitrov Izmirliev (Smirnenski) (1898-1923) est un poète bulgare, un brillant représentant du post-symbolisme dans la littérature bulgare. En dépit d'une mort précoce, il s'est fait remarqué comme un auteur prolifique- l'une des dernières éditions de ses œuvres complètes comprend huit volumes. couvert d'éloges par la critique littérataire de gauche (pricipalement en raison des idées socialistes dans son oeuvre) et considéré par les cercles littéraires conservateurs de son époque comme un auteur de "poésie décorative ",Smirnenski est un poète au remarquable potentiel parodique et doté de possibilités versificatoires exceptionnelles.

27. Baumann, Z. Europe of Strangers - WPTC, 1998, 3; Cohen, R. Transnational Social Movements: an Assessment - WPTC, 1998, 10; Crisen, S. National Identity and Cultural Self-Definition: Modern and Post-modern Romanian Artistic Expression. 1998 (http://www.ics.si.edu/Programs/REGION/ EES); Debeljac, A. Varieties of National Experience: Resistance and Accommodation in Contemporary Slovenian Identity - Space of Identity, 2001, vol. 1, 35-47; Djuric, J. The Concept of Models of Identity: Existance without Identity - In: Golubovif, z. (ed.). Models of Identities in Postcommunist Societies. Yugoslav Philosophical Studies, I. Cultural Heritage and Contemporary Change. Series IVA, Central and Eastern Europe, 1995, vol. 10; Faist, T. Transnationalism in International Migration: Implication for the Study of Citizenship and Culture - WPTC, 1999, 8; Guarnizo, L. Assimilation and Transnationalism. Determinants of Transnational Political Action among Temporary Migrants - AJS, 2003, vol. 108, nom. 3, 1212-1248; Moalem, M. Foreignness and Be/longing: Transnationalism and Immigrant Entrepreneurial Spaces - Comparative Studies of South Asia, Africa and Middle East, 2000, vol. XX, Nos. 1\&2, 200-216; Petrovic, E. Re-Creation of Self: Narrative of Emigrant Women from Yugoslavia living in Western Canada - Space of Identity, 2003, vol. 3, 8-25; Reisenleitner, M. Tradition, Cultural Borders and the Construction of the Space of Identity - Space of Identity, 2001, vol. 1, 7-13; Sandhu, A. No More Inside/Outside: Transnationalism and The New Politic Economy - Alternatives, 2002, vol. 1, nom. 3; Schiffauer, M. Islamism in the Diaspora. The fascination of Political Islam among Second Generation of German Turks - WTPC, 1999, 06; Siemiatycki, M. Immigration, transnationalisme et citoyenneté. Politique et pratique en matière d'immigration au Canada - Cahiers d'études sur la Méditerranee orientale et le monde turco-iranien, 1992, vol 13, janvier-juin; Sklair, L. Transnational Practices 
and the Analyses of Global System. Seminar delivered for the National Communities Programme Seminar Series, 1998, 22; Smith, M., Guarnizo, L. (eds.) Transnationalism from Below Comparative Urban and Community Research, 1998, vol. 6; Vertovic, St. Religion and Diaspora WPTC, 2001, 1; Vertovic, St. Transnational Challenges to the new "Multiculturalism" - WPTC, 2001, 6. Pour une analyse des différentes théories portant sur les phénomènes transnationaux voir Sultanova, R. : «Transnatsionalizma kato podhod pri izuchavaneto na postmigracionnia opit na migrantite ». In: Mihova, M. (dir) Da zhiveesh tam, da sanuvash tuk, p.268-291.

28. Gottmann (J.), La politique des Etats et leur géographie. Paris, 1952, p. 70-71.

29. AEIM, N ${ }^{\circ} 574-$ III, 50

30. "Göçmen olmak, gözü arkada olmak."

31. Parla (Ayse), « Marking Time Across the Bulgarian Turkish Border », Ethnography, 2003, 4(4); Parla (Ayse),. « Locating the Homeland : Bulgarian-Turkish "Return" Migration in Transnational Perspective ", à l'adresse: www.sant.ox.ac.uk/esc/esc-lectures/parla.pdf [consultée le 17 octobre 2008].

32. Tous les interlocuteurs avec l'auteur a eu l'occasion de travailler détenaient la double nationalité.

33. La Bulgarie a rejoint l'Union européenne en janvier 2007 (ndr).

34. Voir Bachelard (G.), La poétique de l'espace, PUF, 1957; Ledru (Raimon), «L'Homme et l'espace ", In Poirier (Jean), Histoire de mœurs. Les temps, l'espace et les rythmes. Paris, 1990, Editions Gallimard, pp. 66-122.

35. Les migrants chassent avant tout des sangliers, une pratique qui est vue comme distinctive ; la chasse constitue à la fois un divertissement et un moyen de se procurer du porc gratuitement. 36. Au sujet des dialectes turcs en Bulgarie voir : Kowalski, (T). Les Turcs et la langue turque de la Bulgarie du Nord-Est. Mémoires de la Commission Orientaliste de l'Académie des Sciences de Cracovie, No. 16. Krakow: Polska Akademja Umiejetnosci, 1933; Andrews, (Alford), Türkiye'de Etnik Gruplar, Ankara, 1992, ANT Yayınları. Les linguistes distinguent plusieurs dialectes turcs de Bulgarie. Ici nous parlons d'un dialecte car il s'agit d'une catégorie d'usage commun, correspondant aux représentations des personnes.

37. Des écoles turques ont existé en Bulgarie sur une longue période, entre l'indépendance du pays obtenue, de fait, en 1878, et même après l'établissement du régime communiste après 1944 . Le Parti communiste bulgare a progressivement adopté une politique visant à réduire leur rôle, avant de décider de les fermer vers la fin des années 1950. Par ailleurs, le pouvoir communiste a créé en 1958 une commission universitaire dont le but était de faire entrer des termes bulgares dans la langue turque. Au terme de cette politique, de nombreux termes turcs, noms de villes, de mois, d'institutions ont été remplacés par des mots bulgares ou translittérés selon des règles linguistiques bulgares. Cette "bulgarisation» du dialecte turc de Bulgarie a contribué à sa distanciation et différenciation par rapport à la langue turque parlée en Turquie. Voir Jalămov (Ibrahim), Istoriya na turskata obshtnost $v$ Bulgariya [Histoire de la communauté turque en Bulgarie], Sofia, 2002, pp. 336-337.

38. AMCIMKV, N³321/19. 02.2004, p. 299.

39. Pour plus de détails sur ces deux derniers groupes de migrants, se reporter à Maeva (Mila), «Les migrants tucs bulgares en République de Turquie (culture et identité). Sofia, IMIR,2006, à l'adresse : www.imir-bg.org/imir/books/balgarskite\%20turci\%20preselnici.pdf 


\section{RÉSUMÉS}

En raison de leur poids démographique, de leurs appartenances ethniques et confessionnelles et de leur distribution géographique, les Turcs bulgares ont toujours fait l'objet d'une attention particulière de la part des pouvoirs publics bulgares et turcs. L'article présent retient toutefois un angle d'approche spécifique en se concentrant sur la trajectoire des Turcs de Bulgarie qui ont quitté le pays pour s'installer en République de Turquie entre 1989 et 2004 . L'article identifie plusieurs types de vagues migratoires: la première, provoquée par le «processus de Renaissance » (l'assimilation forcée), la seconde par les changements politiques et économiques du post-communisme et la troisième, la plus récente, connue sous le nom de «migration de brues ». Il envisage ensuite les difficultés d'adaptation que les Turcs installés en République de Turquie ont pu rencontrer. Parmi les Turcs de Bulgarie, trois sous-groupes peuvent être distingués : les « migrants », les « ré-émigrants » et les « personnes naturalisées ». La politique de l'Etat d'origine et de l'Etat d'accueil des migrants, les modes d'organisation du nouveau territoire, les stratégies d'adaptation sociale, le niveau de réalisation socio-économique, les relations de contact et de conflit avec la population locale exercent tous une influence sur les auto-définitions ethnoculturelles adoptées par les migrants turcs en Turquie. La notion de " communauté transétatique " nous semble ici précieuse pour désigner une communauté qui appartient à deux Etats et se déplace régulièrement de l'un à l'autre dans un effort pour préserver son ancien modèle culturel tout en cherchant à optimiser ses conditions de vie. Les principales caractéristiques de ce groupe sont la double citoyenneté, une mobilité permanente, un habitat compact, des activités professionnelles communes, la diglossie, le bilinguisme, la préservation de la religion et de la culture nationale traditionnelle, l'endogamie et, finalement, la valorisation d'un système de valeurs propre.

\section{INDEX}

Index géographique : Bulgarie, Turquie

Mots-clés : Turcs, Minorité turque, Migrations, émigration

\section{AUTEURS}

\section{MILA MAEVA}

Dr. Mila Maeva est chercheur à l'Institut d'ethnographie de l'Académie des Sciences bulgares (BAN), Sofia. Diplômée d'ethnologie à l'Université de Sofia « Kliment Ohridski », elle y a soutenu en 2005 une thèse sur " l'Identité ethnoculturelle des Turcs bulgares migrants ", après avoir également effectué des recherches sur les Bulgares musulmans dans le cadre d'un travail de maîtrise. En 2007, elle a obtenu une bourse de spécialisation du British Council au Center for Research of Ethnic Relations (CRER) de l'Université de Warwick (Grande-Bretagne). Ses recherches actuelles portent sur les migrations bulgares en Grande-Bretagne, mais également sur les enjeux identitaires et les groupes ethnoculturels (musulmans, turcs). Elle a récemment publié : « Religija i identičnost na turcite, preselnici ot Bălgarija v Republika turcija [Religion et identités des Turcs émigrants en République de Turquie], in : Margarita Karamihova (dir.), Ti imaš ajsen znak! 'Zavrăštane' na religiosnostta $v$ kraja na XX i načaloto na XXI vek [Tu as un signe distinctif clair ! Le « retour » de la religiosité à la fin du XXème et au début du XXIème siècle], Sofia : Faber 
OOD, 2007, p.143-158; Bălgarskite turci-preselnici v Republika Turcija (kultura i identičnost) [Les Turcs bulgares émigrants en République de Turquie (culture et identité)], Sofia : IMIR, 2006.

mmila_maeva@yahoo.co.uk 